\title{
Research
}

\section{Mutational signatures of DNA mismatch repair deficiency in C. elegans and human cancers}

\author{
Bettina Meier, ${ }^{1,7}$ Nadezda V. Volkova, ${ }^{2,7}$ Ye Hong, ${ }^{1}$ Pieta Schofield, ${ }^{1,3}$ \\ Peter J. Campbell, ${ }^{4,5,6}$ Moritz Gerstung, $^{2}$ and Anton Gartner ${ }^{1}$ \\ ${ }^{1}$ Centre for Gene Regulation and Expression, University of Dundee, Dundee DD1 5EH, United Kingdom; ${ }^{2}$ European Molecular Biology \\ Laboratory, European Bioinformatics Institute (EMBL-EBI), Hinxton CB10 1SD, United Kingdom; ${ }^{3}$ Division of Computational Biology, \\ University of Dundee, Dundee DD1 5EH, United Kingdom; ${ }^{4}$ Cancer Genome Project, Wellcome Trust Sanger Institute, Hinxton, \\ Cambridge CB10 1SA, United Kingdom; ${ }^{5}$ Department of Haematology, University of Cambridge, Cambridge CB2 OXY, \\ United Kingdom; ${ }^{6}$ Department of Haematology, Addenbrooke's Hospital, Cambridge CB2 OXY, United Kingdom
}

\begin{abstract}
Throughout their lifetime, cells are subject to extrinsic and intrinsic mutational processes leaving behind characteristic signatures in the genome. DNA mismatch repair (MMR) deficiency leads to hypermutation and is found in different cancer types. Although it is possible to associate mutational signatures extracted from human cancers with possible mutational processes, the exact causation is often unknown. Here, we use $C$. elegans genome sequencing of pms- 2 and $m / h-1$ knockouts to reveal the mutational patterns linked to $C$. elegans MMR deficiency and their dependency on endogenous replication errors and errors caused by deletion of the polymerase $\varepsilon$ subunit pole-4. Signature extraction from 215 human colorectal and 289 gastric adenocarcinomas revealed three MMR-associated signatures, one of which closely resembles the $C$. elegans MMR spectrum and strongly discriminates microsatellite stable and unstable tumors (AUC $=98 \%$ ). A characteristic difference between human and C. elegans MMR deficiency is the lack of elevated levels of NCG > NTG mutations in C. elegans, likely caused by the absence of cytosine $(\mathrm{CpG})$ methylation in worms. The other two human MMR signatures may reflect the interaction between MMR deficiency and other mutagenic processes, but their exact cause remains unknown. In summary, combining information from genetically defined models and cancer samples allows for better aligning mutational signatures to causal mutagenic processes.
\end{abstract}

[Supplemental material is available for this article.]

Cancer is a genetic disease associated with the accumulation of mutations. A major challenge is to understand mutagenic processes acting in cancer cells. Accurate DNA replication and the repair of DNA damage are important for genome maintenance. The identification of cancer predisposition syndromes caused by defects in DNA repair genes was important to link the etiology of cancer to increased mutagenesis. One of the first DNA repair pathways associated with cancer predisposition was DNA mismatch repair (MMR). MMR corrects mistakes that arise during DNA replication. Mutations in MMR genes are associated with hereditary nonpolyposis colorectal cancer (HNPCC), also referred to as Lynch Syndrome (Fishel et al. 1993; Bronner et al. 1994; Nicolaides et al. 1994; Papadopoulos et al. 1994; Miyaki et al. 1997).

DNA mismatch repair is initiated by the recognition of replication errors by MutS proteins, initially defined in bacteria. In $S$. cerevisiae and mammalian cells, two MutS complexes termed MutS $\alpha$ and MutS $\beta$, comprised of MSH2/MSH6 and MSH2/MSH3 heterodimers, respectively, are required for DNA damage recognition albeit with differing substrate specificity (Drummond et al. 1995; Habraken et al. 1996; Genschel et al. 1998). Binding of MutS to the DNA lesion facilitates subsequent recruitment of the MutL complex. MutL enhances mismatch recognition and promotes a conformational change in MutS to allow for the sliding

\footnotetext{
${ }^{7}$ These authors contributed equally to this work. Corresponding authors: a.gartner@dundee.ac.uk, moritz.gerstung@ebi.ac.uk

Article published online before print. Article, supplemental material, and publication date are at http://www.genome.org/cgi/doi/10.1101/gr.226845.117. Freely available online through the Genome Research Open Access option.
}

of the MutL/MutS complex away from mismatched DNA (Allen et al. 1997; Gradia et al. 1999). DNA repair is initiated in most systems by a single-stranded nick generated by MutL (MutH in E. coli) on the nascent DNA strand at some distance to the lesion (Kadyrov et al. 2006, 2007). Exonucleolytic activities in part conferred by Exo1 contribute to the removal of the DNA stretch containing the mismatch followed by gap filling via lagging strand DNA synthesis (Goellner et al. 2015). The most prominent MutL activity in human cells is provided by the MutL $\alpha$ heterodimer MLH1/PMS2 (Prolla et al. 1998; Cannavo et al. 2005). Moreover, human MLH1 is found in heterodimers with PMS1 and MLH3, called MutL $\beta$ and MutL $\gamma$. Of these, only MutL $\gamma$ is thought to have a minor role in MMR (Cannavo et al. 2005). The C. elegans genome does not encode obvious MutL $\beta$ and $\gamma$ subunits (PMS1 and MLH3 homologs, respectively), whereas the MutL $\alpha$ subunits MLH-1 and PMS-2 can be readily identified using homology searches (Supplemental Table S1).

Analysis of mutations in microsatellite loci of MLH1-deficient colorectal cancer cell lines suggested rates of repeat expansion or contraction between $8.4 \times 10^{-3}$ and $3.8 \times 10^{-2}$ per locus and generation (Bhattacharyya et al. 1994; Hanford et al. 1998). Estimates using $S$. cerevisiae revealed a 100 - to 700 -fold increase in DNA repeat tract instability in pms 2 , mlh1, and $m s h 2$ mutants (Strand et al. 1993) and an approximate fivefold increase in base substitution rates (Yang et al. 1999). C. elegans assays using reporter systems or selected, PCR-amplified regions revealed a $>30$-fold

C) 2018 Meier et al. This article, published in Genome Research, is available under a Creative Commons License (Attribution 4.0 International), as described at http://creativecommons.org/licenses/by/4.0/. 
increased frequency of single base substitutions in $m s h-6$, a 500fold increase in mutations in A/T homopolymer runs, and a 100fold increase in mutations in dinucleotide repeats (Degtyareva et al. 2002; Tijsterman et al. 2002; Denver et al. 2005), akin to the frequencies observed in yeast and mammalian cells (Strand et al. 1993; Hanford et al. 1998). Recently, whole-genome sequencing approaches using diploid $S$. cerevisiae started to provide a genome-wide view of MMR deficiency. S. cerevisiae lines carrying an $m s h 2$ deletion alone or in conjunction with point mutations

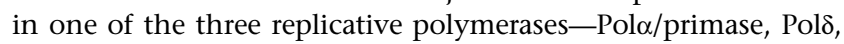
and Pols-were propagated over multiple generations to determine the individual contribution of replicative polymerases and MMR to replication fidelity (Lang et al. 2013; Lujan et al. 2014, 2015). These analyses estimated an average mutation rate of $1.6 \times 10^{-8}$ per base pair per generation in msh 2 mutants and a further increased rate in double mutants of $m s h 2$ and any of the replicative polymerases (Lujan et al. 2014, 2015). A synergistic increase in mutagenesis was also recently observed in childhood tumors in which MMR deficiency and mutations in replicative polymerase $\varepsilon$ and $\delta$, required for leading and lagging strand DNA synthesis, respectively, occurred (Shlien et al. 2015).

In human cancer samples, 30 mutational signatures (referred to as COSMIC signatures from here on) have been uncovered by mathematical modeling across a large number of cancer genomes representing more than 30 tumor types (http://cancer.sanger.ac. $\mathrm{uk} /$ cosmic/signatures) (Alexandrov et al. 2013a,b). These signatures are largely defined by the relative frequency of the six possible base substitutions $(\mathrm{C}>\mathrm{A}, \mathrm{C}>\mathrm{G}, \mathrm{C}>\mathrm{T}, \mathrm{T}>\mathrm{A}, \mathrm{T}>\mathrm{C}$, and $\mathrm{T}>\mathrm{G})$ in the sequence context of their adjacent $5^{\prime}$ and $3^{\prime}$ base. Of these, COSMIC signatures 6, 15, 20, 21, and 26 have been associated with MMR deficiency with several MMR signatures being present in the same tumor sample (Alexandrov et al. 2013a,b). It is not clear whether these MMR signatures are conserved across evolution and how they reflect MMR defects. Therefore, MMR signatures deduced from defined monogenic MMR-defective backgrounds (which we will herein refer to as mutational patterns) could contribute to the refinement of computationally derived mutational signatures extracted from cancer genomes.

Here, we investigate the genome-wide mutational impact of the loss of the MutL mismatch repair genes mlh-1 and pms-2 in the nematode C.elegans. Furthermore, we address the contribution of a deletion of pole-4, a nonessential accessory subunit of the leading-strand DNA polymerase Pole, to mutation profiles and hypermutation.

\section{Results}

\section{Mutation rates and profiles of $m / h-1, p m s-2$, and pole-4 single} mutants grown over 20 generations

We previously established C. elegans mutation accumulation assays and demonstrated that defects in major DNA damage response and DNA repair pathways, including nucleotide excision repair, base excision repair, DNA crosslink repair, DNA end-joining, and apoptosis, did not lead to overtly increased mutation rates when lines were propagated for 20 generations (Meier et al. 2014). The experimental setup takes advantage of the 3-4 d life cycle of $C$. elegans and its hermaphroditic reproduction by self-fertilization. This allows for the propagation of clonal C. elegans lines, which in each generation pass through a single-cell bottleneck provided by the zygote. We now extend these studies to MMR deficiency conferred by MutL $\alpha$ mutations mlh-1 and pms-2. Because null al- leles of the human and C. elegans leading-strand polymerase Pole catalytic subunit, POLE and pole-1, respectively, cause lethality, we focused our analysis on a nonessential C. elegans Pole subunit, termed POLE-4. Dbp3p, the $S$. cerevisiae POLE-4 ortholog, has been implicated in stabilizing POLE interaction with the primer-template DNA complex (Aksenova et al. 2010).

We detected an average of four base substitutions and two insertions/deletions in wild-type C. elegans lines propagated for 20 generations (Fig. 1A,B). In contrast, mlh-1 and pms-2 mismatch repair single mutants carried an average of 1174 and 1191 unique mutations, respectively, of which 288 and 309 were base substitutions (Fig. 1A) and 886 and 882 indels, defined as small insertions and deletions of $<400 \mathrm{bp}$ (Fig. 1B). The nature of single nucleotide changes and the overall mutation burden were congruent across independent lines of the same genotype, and mutation numbers linearly increased from F10 to F20 generation lines (Fig. 1). In contrast to $m l h-1$ and pms-2, pole-4 mutants exhibited mutation numbers and profiles not significantly different from wild-type (Fig. 1; Supplemental Table S2).

\section{Mutation rates and patterns in pole-4; pms-2 double mutants}

To further investigate the role of pole- 4 and the genetic interaction with MMR deficiency, we generated pole-4; pms-2 double mutants. pms-2 mutants carried an average of 145 base substitution and 527 indels over 10 generations, roughly half the number we observed in the F20 generation (Fig. 1C,D; Supplemental Table S2). In comparison, the number of single base substitutions and indels was increased $\sim 4$.4-fold and $\sim 1$.4-fold in pole-4; pms-2 double mutants to an average of 637 and 723, respectively (Fig. 1C,D; Supplemental Table S2). We did not identify any structural variants (SVs) in the genotypes analyzed except for pole-4, in which a single SV was observed in three F10 mutation accumulation lines (Supplemental Table S2). We could not readily propagate pole-4; pms-2 beyond the F10 generation, suggesting that a mutation burden higher than 500-700 single base substitutions (Fig. 1C) in conjunction with 700-750 indels (Fig. 1D) might be incompatible with organismal reproduction. The increased mutation burden of pole-4; pms-2 double mutants compared to that of $p m s-2$ and to the wild-type mutation rate of pole- 4 suggests that replication errors occur at increased frequency in the absence of pole- 4 but are effectively repaired by MMR.

Assuming that it takes 15 cell divisions to go through the $C$. elegans life cycle and considering that heterozygous mutations can be lost during self-fertilization (Methods), we calculated a mutation rate per base pair and germ cell division of $1.0 \times 10^{-9}(95 \%$ CI: $7.96 \times 10^{-10}$ to $1.25 \times 10^{-9}$ ) for wild-type and $1.19 \times 10^{-9}$ ( $95 \%$ CI: $9.58 \times 10^{-10}$ to $1.45 \times 10^{-9}$ ) for pole- 4 mutants. In contrast, mutation rates for $m l h-1$ and pms- 2 were $7.10 \times 10^{-8}(95 \%$ CI: $6.86 \times 10^{-8}$ to $\left.7.33 \times 10^{-8}\right)$ and $7.28 \times 10^{-8}(95 \% \mathrm{CI}: 7.10 \times$ $10^{-8}$ to $\left.7.48 \times 10^{-8}\right)$, respectively. pole-4; pms-2 double mutants exhibited a mutation rate of $1.51 \times 10^{-7}\left(95 \% \mathrm{CI}: 1.45 \times 10^{-7}\right.$ to $1.56 \times 10^{-7}$ ).

The genome-wide mutation rates observed in the absence of C. elegans MutL $\alpha$ proteins MLH- 1 and PMS- 2 are in line with mutation rates previously determined for C. elegans MutS and S. cerevisiae MMR mutants (Strand et al. 1993; Yang et al. 1999; Degtyareva et al. 2002; Tijsterman et al. 2002; Denver et al. 2005). However, unlike in mammalian cells (Yao et al. 1999; Baross-Francis et al. 2001), C. elegans mlh-1 and pms-2 mutants exhibited almost identical mutation rates and profiles, suggesting that the inactivation of the MutL $\alpha$ heterodimer is sufficient to 

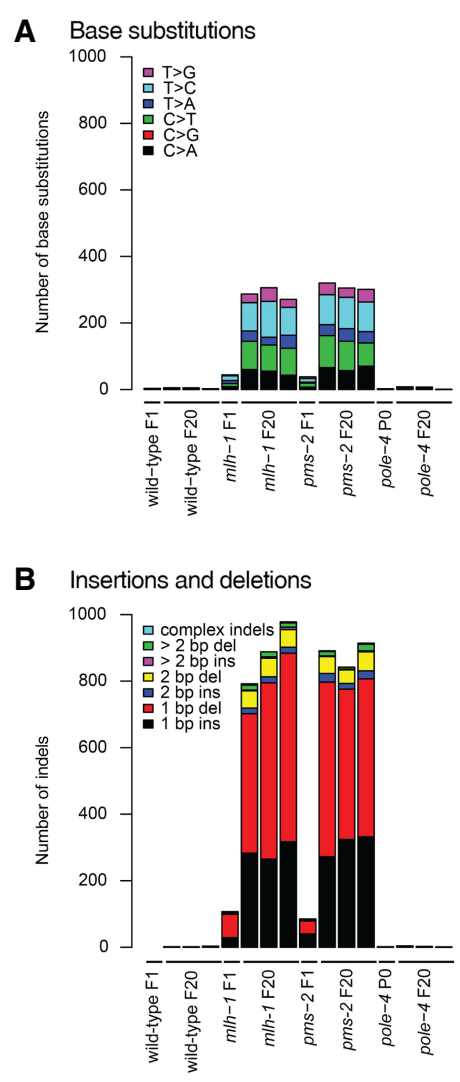

D

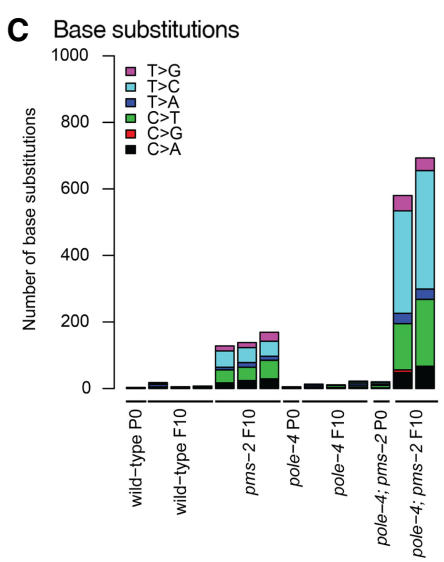

D Insertions and deletions

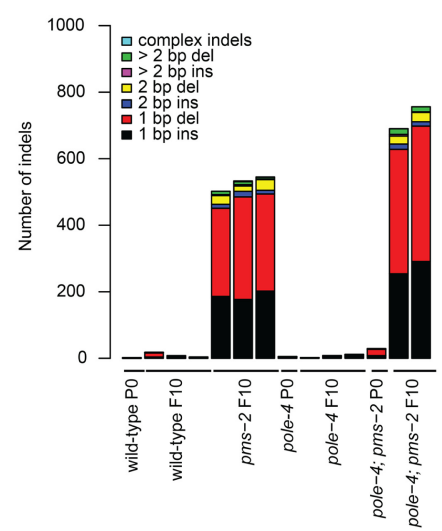

E Base substitution spectra

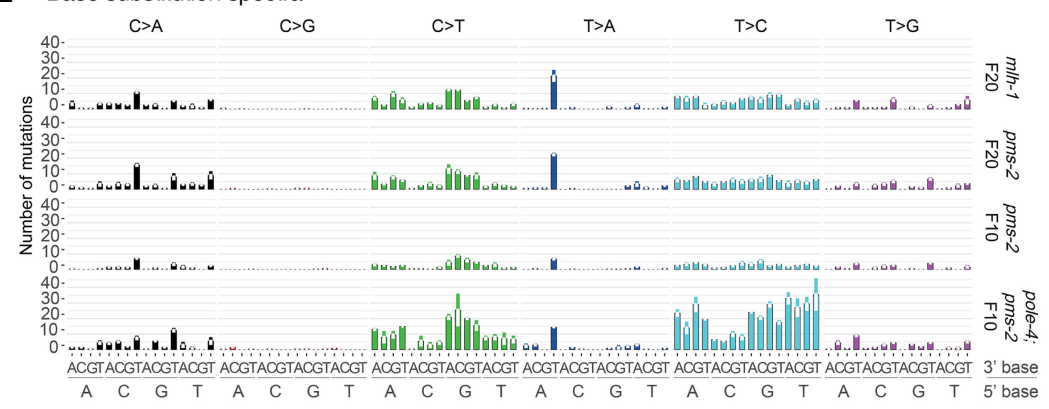

$\mathbf{F}$

Sequence context embedding small indels
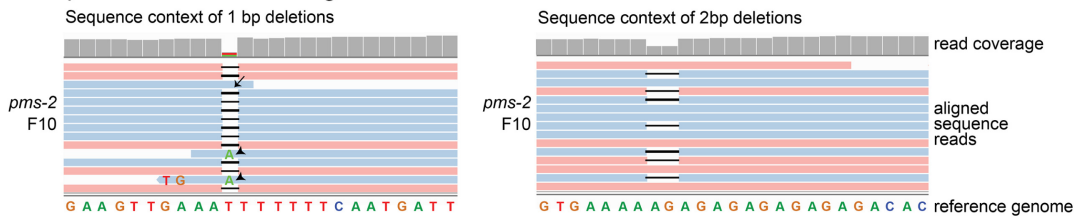

Figure 1. Mutations in C. elegans wild-type and MMR mutants grown for 10 or 20 generations. Identical base substitutions as well as indels occurring in the same genomic location among samples of the entire data set (duplicates) were excluded from the analysis, thus only reporting mutations unique to each individual sample. (A) Number and types of base substitutions identified in the parental ( $\mathrm{PO}$ ) or one first generation (F1) line and three independently propagated F20 lines of wild-type, $m / h-1, p m s-2$, and pole-4 single mutants. (B) Number and types of insertions and deletions (indels) identified in initial (P0 or F1) and three independently propagated F20 lines of wild-type, $m / h-1, p m s-2$, and pole-4 single mutants. (C) Number and types of base substitutions observed in the parental (PO) line and 2-3 independently propagated F10 lines of wild-type, pms-2 and pole-4 single, and pole-4; pms-2 double mutants. (D) Number and type of indels observed in the parental (P0) and 2-3 independently propagated F10 lines of wild-type, pms-2 and pole-4 single, and pole-4; pms-2 double mutants. (E) Average number of base substitutions identified across all individual lines per genotype in their $5^{\prime}$ and $3^{\prime}$ base sequence context in $m / h-1$ and pms- 2 single and in pms-2 single and pole-4; pms-2 double mutants. Error bars represent the standard error of the mean. (F) Examples of indel sequence contexts. Sequence reads aligned to the reference genome WBcel235.74 visualized in Integrative Genomics Viewer (Robinson et al. 2011). A 1-bp (left) and a 2-bp deletion (right) are shown. A subset of sequence reads, which end close to an indel, erroneously aligned across the indel resulting either in wild-type bases (arrow) or base changes (arrowheads). Such wrongly called base substitutions were removed during filtering (Methods) using the deepSNV package (Gerstung et al. 2012, 2014). induce a fully penetrant MMR phenotype consistent with the absence of PMS1 MutL $\beta$ and MLH3 MutL $\gamma$ homologs in C. elegans. Our finding that pole-4 mutants do not show increased mutation rates is surprising given that the deletion of the budding yeast pole- 4 homolog DPB3 leads to mutation rates comparable to the proofreading-deficient pol2-4 allele of the Pole catalytic subunit (Aksenova et al. 2010; Lujan et al. 2012). Increased mutation rates have also been reported for proofreading mutants of the Pole catalytic subunit in mice and human, and in humans such mutations are associated with an increased predisposition to colorectal cancer (Albertson et al. 2009; Lujan et al. 2012; Palles et al. 2013).

\section{Distribution and sequence context of base substitutions}

We next wished to determine the mutational patterns associated with DNA mismatch repair defects alone and combined with pole- 4 deficiency. $\mathrm{T}>\mathrm{C}$ and $\mathrm{C}>\mathrm{T}$ transitions were present more frequently than $\mathrm{T}>\mathrm{A}, \mathrm{T}>\mathrm{G}, \mathrm{C}>\mathrm{A}$, and $\mathrm{C}>\mathrm{G}$ transversions in mlh-1 and pms-2 single and pole-4; pms-2 double mutants (Fig. 1A,C; Supplemental Table S2). A similar preponderance of $\mathrm{T}>\mathrm{C}$ and $\mathrm{C}>\mathrm{T}$ transitions was previously observed in $S$. cerevisiae msh2 mutants and in MMR-defective human cancer lines (Alexandrov et al. 2013a; Lujan et al. 2014; Supek and Lehner 2015). Analyzing all base substitutions within their $5^{\prime}$ and $3^{\prime}$ sequence context, we found no prominent enrichment of distinct $5^{\prime}$ and $3^{\prime}$ bases associated with $\mathrm{T}>\mathrm{C}$ transitions in mlh-1 and pms- 2 single mutants. In contrast, $\mathrm{T}>\mathrm{A}$ transversions occurred with increased frequency in an ATT context, $\mathrm{C}>\mathrm{T}$ transitions in a GCN context, and $\mathrm{C}>\mathrm{A}$ transversions in a N CT context (Fig. 1E).

Interestingly, $>90 \%$ of $\mathrm{T}>\mathrm{A}$ transversions in an ATT context occurred in homopolymer runs; the majority $(>75 \%)$ in the context of two adjoining $\mathrm{A}$ and $\mathrm{T}$ homopolymers (Supplemental Fig. S1A). An increased frequency of base substitution at the junction of adjacent repeats has also been reported in S. cerevisiae MMR mutants, giving rise to the speculation that such base substitutions may be generated by double slippage events (Lang et al. 2013). Moreover, we observed several examples in which one or several base substitutions had occurred that converted a repeat sequence such that it 
became identical to flanking repeats consistent with polymerase slippage across an entire repeat (Supplemental Fig. S1B-D). Such mechanisms could lead to the equalization of microsatellite repeats, a phenomenon referred to as microsatellite purification (Harr et al. 2000).

Although we could not define mutational patterns specifically associated with pole- 4 loss due to the low number of mutations, the profile of pole-4; pms-2 double mutants differed from MMR single mutants. In addition to $\mathrm{C}>\mathrm{T}$ transitions in a GCN context, $\mathrm{T}>\mathrm{C}$ transitions were generated with higher frequency, accounting for $>50 \%$ of all base changes (Fig. 1C). Among these, $\mathrm{T}>\mathrm{C}$ substitutions in the context of a flanking $5^{\prime}$ cytosine were underrepresented (Fig. 1E). A higher proportion of $\mathrm{T}>\mathrm{C}$ changes, not embedded in a defined sequence context, has been reported for MMR-deficient tumor samples containing mutations in the lagging strand polymerase Polo (Shlien et al. 2015), but not in S. cerevisiae and human tumors with a combined MMR and Pole deficiency (Lujan et al. 2014; Shlien et al. 2015). No obvious chromosomal clustering of base substitutions was observed in pms-2 and pole-4; pms-2 grown for 10 generations (Supplemental Fig. S2A).

\section{Sequence context of insertions and deletions associated with MMR deficiency}

The majority of mutations observed in $m l h-1$ and pms- 2 single and pole-4; pms-2 double mutants were small insertions/deletions (indels) (Fig. 1B,D). Of these, $\sim 90 \%$ constituted 1-bp indels (Fig. 1B,D; Supplemental Table S2) with most 1-bp indels occurring in homopolymer runs (Figs. 1F, 2B). On average, 2-bp indels accounted for $5.5 \%-8.6 \%$ of indels observed (Fig. 1B,D; Supplemental Table S2) and affected dinucleotide repeat sequences (Fig. 1F) and homopolymer runs at similar frequency, as recently also
A

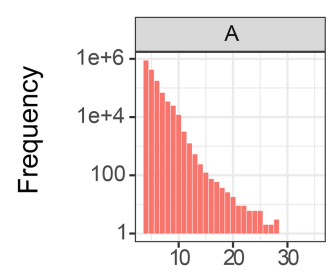

Homopolymer frequency in C. elegans genome

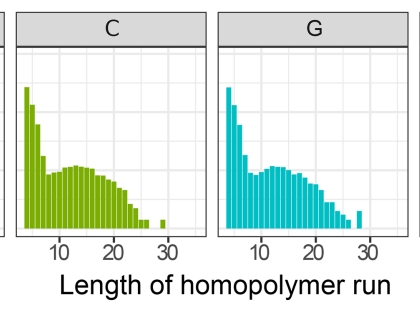

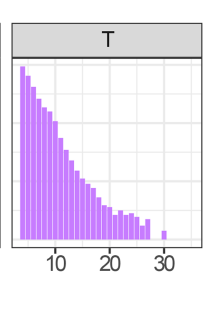

Homopolymer distribution by base

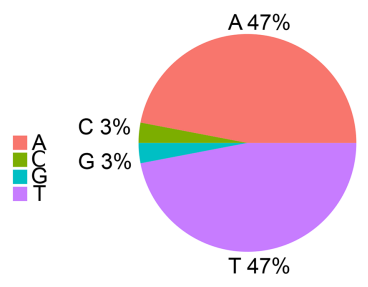

B Frequency of $1 \mathrm{bp}$ indels in homopolymers

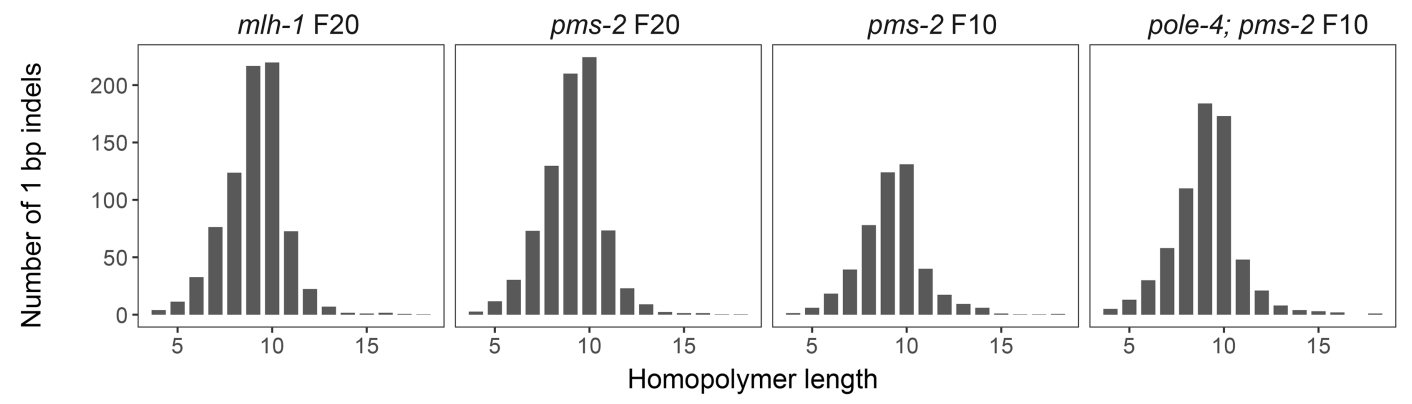

C GAM fit for ratio of $1 \mathrm{bp}$ indels per homopolymer
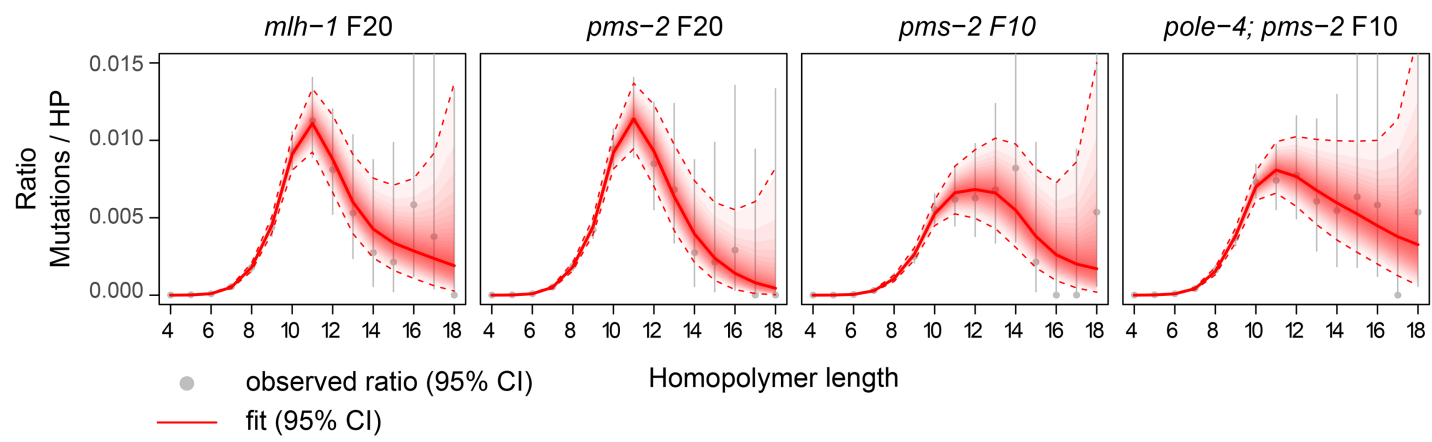

Homopolymer length

Figure 2. Correlation between homopolymer length and the frequency of $+1 /-1$ bp indels. ( $A$ ) Distribution of homopolymer repeats encoded in the $C$. elegans genome by length and DNA base shown in $\log _{10}$ scale (left) and the relative percentage of $A, C, G$, and T homopolymers in the genome (right). (B) Average number of 1-bp indels in homopolymer runs for $m / h-1 \mathrm{~F} 20$, pms-2 F20, pms-2 F10, and pole-4; pms-2 F10 mutant lines by homopolymer length . (C) Generalized additive spline model (GAM) fit for the ratio of 1-bp indels normalized to the frequency of homopolymers (HPs) in the genome. The average frequency observed across three lines is depicted as a gray dot; gray bars indicate the $95 \%$ confidence interval. The red line indicates best fit. Red dotted lines represent the corresponding $95 \%$ confidence interval. 
reported for MMR mutants in S. cerevisiae (Lujan et al. 2015). Trinucleotide repeat instability is associated with a number of neurodegenerative disorders, such as fragile X syndrome, Huntington's disease, and Spinocerebellar Ataxias (Brouwer et al. 2009). Based on our analysis, trinucleotide repeat sequences are present in the C. elegans genome at a $>400$-fold lower frequency than homopolymer runs (Methods; Supplemental Data Analysis). We observed between three and seven trinucleotide indels per 10 generations in mlh-1 and pms-2 mutants (Supplemental Table S2). However, these occurred predominantly in homopolymer sequences precluding an estimation of mutation rates for trinucleotide repeats.

Clustering of 1-bp indels was not evident for pms-2 and pole-4; pms-2 F10 lines beyond a somewhat reduced occurrence in the center of $C$. elegans autosomes, which correlates with reduced homopolymer frequency in these regions (Supplemental Fig. S2B).

\section{Dependency of 1-bp indel frequency on homopolymer length}

Given the high number of indels present in homopolymer repeats, we aimed to investigate the correlation between indel frequency and homopolymer length. Overall, we identified 3,433,785 homopolymers in the $C$. elegans genome, ranging in length between 4 and 35 nt (Fig. 2A; Methods). Of all homopolymers, 47\% each were poly $(\mathrm{A})$ or poly $(\mathrm{T})$ repeats, their frequencies decreasing with increasing length (Fig. 2A). C and $\mathrm{G}$ each comprised 3\% of all homopolymers with frequencies decreasing up to a length of 8 $\mathrm{bp}$, plateauing between 8 and $17 \mathrm{bp}$, and further decreasing for longer homopolymer tracts (Fig. 2A). These findings are consistent with a previous report on homopolymers $>7$ bp long (Denver et al. 2004). In C. elegans MMR mutant backgrounds, the frequency of 1bp indels increased with homopolymer length of up to 9-10 bp and trailed off in longer homopolymers (Fig. 2B). Because the frequency of homopolymer tracts decreases with length (Fig. $2 \mathrm{~A})$, we normalized for homopolymer number. To incorporate the uncertainty of the frequency estimation, we applied an additive model (Methods), which supported our findings of a rapid increase in indel frequency in homopolymers up to a repeat length of 10 bases, followed by a drop or plateau in indel frequency for longer homopolymers with decreasing confidence (Fig. 2C). Firm conclusions about indel frequencies in homopolymers $>13 \mathrm{bp}$ are precluded by the lack of statistical power due to the low numbers of both long homopolymers in the genome and associated indels observed (Fig. 2A,B). In summary, our data suggest that replicative polymerase slippage occurs more frequently with increasing homopolymer length, with a peak for homopolymers of 10-11 nt, followed by reduced slippage frequency in longer homopolymers. These results are consistent with observations in budding yeast (Lang et al. 2013) and a recent study using human MLH1 KO organoids (Drost et al. 2017).

\section{Comparison of $C$. elegans MMR patterns to MMR signatures derived from human colorectal and gastric adenocarcinoma samples}

To assess how our findings relate to mutation profiles occurring in human cancer, we analyzed whole-exome sequencing data from the TCGA colorectal adenocarcinoma project COAD-US (http://icgc.org/icgc/cgp/73/509/1134) (The Cancer Genome Atlas Network 2012) and the TCGA gastric adenocarcinoma project STAD-US (https://icgc.org/icgc/cgp/69/509/70268) (The Cancer Genome Atlas Research Network 2014). These cancer types are commonly associated with MMR deficiency. These data sets con- tain single nucleotide (SNV) and indel variant calls from 215 and 289 donors, respectively. Having observed high 1-bp indel frequencies associated with homopolymer repeats in C. elegans pms-2 and mlh-1 mutants (Figs. 1B,D, 2B), we also considered indels in our analysis of human mutational signatures.

A $t$-distributed stochastic neighbor embedding ( $t$-SNE) representation of the cosine similarities of mutation spectra revealed a distinctive grouping of cancer samples (Fig. 3A; Methods). De novo signature extraction across both tumor cohorts combined revealed eight main mutational signatures (Fig. 3B; Methods). Comparing these to existing COSMIC signatures by calculating the similarity score between their base substitution profiles showed that many had a counterpart in the COSMIC database with high similarity (Table 1; Supplemental Fig. S3B), validating our results. We labeled three of the de novo signatures as "MMR-1-3." MMR-1 shares similarity with COSMIC signature 20, MMR-2 with COSMIC signature 15 , and MMR-3 with COSMIC signatures 21 and 26 (Table 1; Supplemental Fig. S3B).

One signature identified in the tumor samples showed characteristics of POLE mutations ("POLE"; http://cancer.sanger.ac. uk/cosmic/signatures) (Fig. 3B; Supplemental Fig. S3B; Alexandrov et al. 2013a). C > T mutations in a CpG base context result from $5 \mathrm{meC}$ deamination and are referred to as "Clock-1" (Fig. 3B; Supplemental Fig. S3B). "Clock-2" is present in the majority of samples and likely reflects background mutation rates (Fig. 3A,B). Signature "17-like" is of unknown etiology, predominantly found in stomach cancers, and related to COSMIC signature 17 (Fig. 3B; Supplemental Fig. S3B). Finally, we identified a signature indicative of SNP contamination characterized by a high overlap of somatic mutations with SNPs present in the human population and a lower nonsynonymous to synonymous ratio, as expected for germline variants (Fig. 3B; Supplemental Material).

To confirm the link between signatures MMR-1-3 and mismatch repair deficiency, we correlated their occurrence with the microsatellite stability status of samples according to the Stanford TCGA Clinical Explorer (http://genomeportal.stanford. edu/pan-tcga).

We considered the samples with microsatellite instable-high (MSI-H) status as MMR-deficient and refer to them as microsatellite instable (MSI), whereas microsatellite instable-low (MSI-L) and microsatellite stable (MSS) samples are considered MMR-proficient and referred to as MSS. In the COAD cohort, 40 of the 215 (19\%) samples, and in the STAD, 63 of the $289(22 \%)$ samples were assigned MSI status. Comparing MSI/MMR-deficient and MSS/ MMR-proficient samples, we found that the fraction of MMR-1, MMR-2, and MMR-3 signatures was strongly enriched in a cluster of microsatellite instable cancer samples (Wilcoxon rank-sum test $P$-values $1.7 \times 10^{-51}, 2.7 \times 10^{-22}$, and $9.8 \times 10^{-29}$, respectively) (Fig. 3A, top left, 3C). Consistent with the results from C. elegans, $82 \%$ of indels in the human cancer samples were 1-bp insertions or deletions (25,093 and 30,561 in COAD and STAD cohort, respectively). The majority of these occurred in homopolymer runs at frequencies ranging between $69 \%$ and $72 \%$ in COAD and $91 \%$ and 93\% in STAD samples (Supplemental Fig. S4B). Of all signatures identified, only MMR-1 highly correlated with the amount of 1bp indels (Pearson correlation coefficient of 0.99), showing a stable trend compared to other MMR signatures (Fig. 3B,D). MMR-1 signature contribution was a remarkably accurate indicator of MSI/MMR deficiency (AUC of $0.98,95 \%$ confidence interval [CI] 0.97-1).

Individual signatures often represent the most extremes of the mutational spectrum; a typical tumor, however, is usually represented by a linear combination of multiple processes. In the

\section{Genome Research}

www.genome.org 
A Similarity map of mutational profiles in colorectal and stomach adenocarcinoma

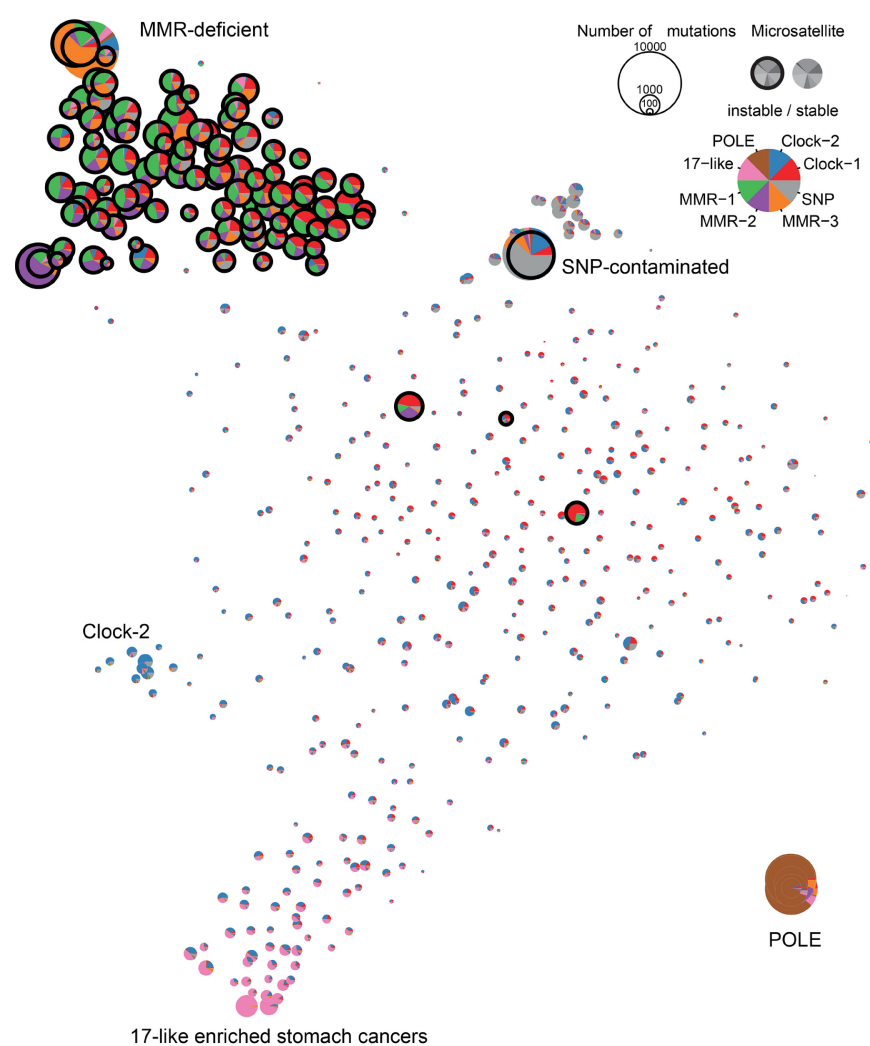

B Extracted mutational signatures

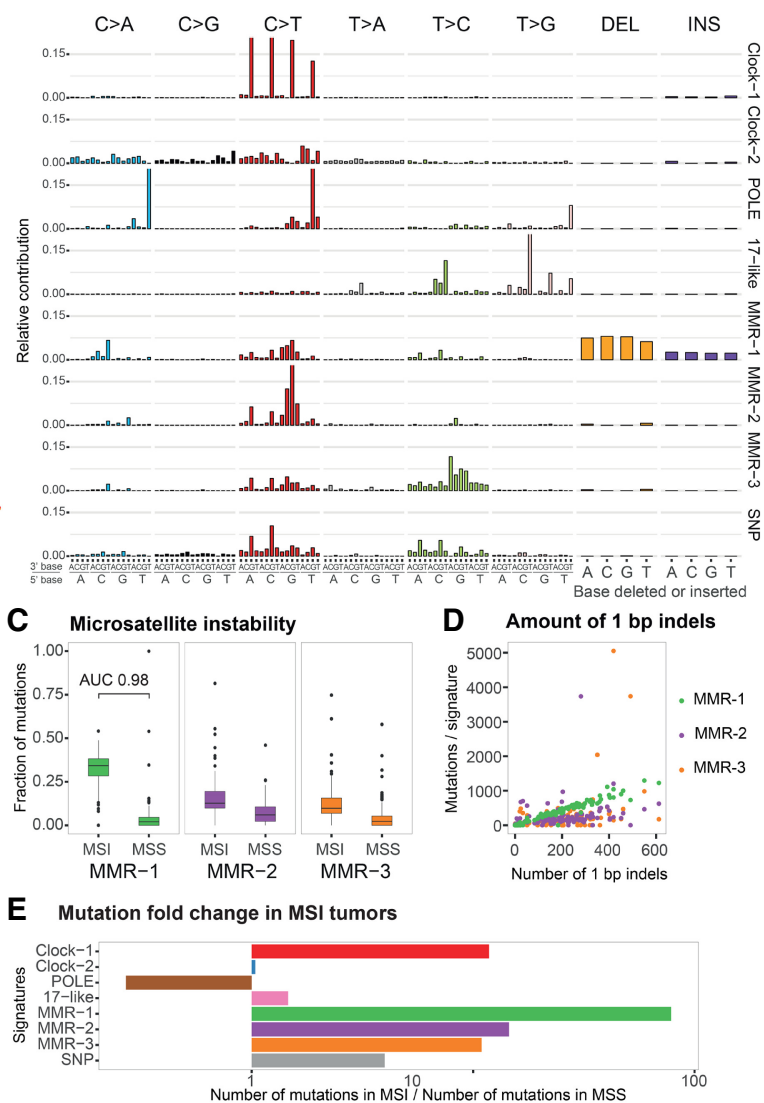

Figure 3. Identification of de novo signatures from human colorectal and gastric adenocarcinoma samples (COAD-US and STAD-US projects) and their contribution to samples clinically classified as microsatellite instable (MSI) or microsatellite stable (MSS). (A) Two-dimensional representation of the mutational profile composition across cancer samples. The size of each circle reflects the mutation burden. MSI samples are highlighted by a bold, black outline. The color of segments reflects the signature composition. (B) Mutational signatures including base substitutions and 1-bp indels derived from the combined COAD-US and STAD-US data sets. (C) Relative contribution of MMR-1, MMR-2, and MMR-3 signatures to cancer samples clinically classified as MSI or MSS. Box plot with outliers shown as individual filled circles. Area under the curve (AUC) value for MMR-1 contribution indicates the probability of a random MSI sample having higher MMR-1 contribution than a random MSS sample. (D) Number of mutations assigned to signatures MMR-1 (green), MMR-2 (purple), and MMR-3 (orange) plotted against the number of 1-bp indels in the same sample. (E) Fold change in the average number of mutations assigned to different signatures in MSI samples compared to MSS samples. As expected, the number of POLE-related mutations is higher in MSS samples as all POLE-deficient tumors are MSS. Apart from MMR signatures, Clock-1 signature also contributes over 10 times more mutations to MSI samples than to MSS. SNP associated mutations are likely due to unfiltered SNPs that are prevalent in the human population (Supplemental Material).

cluster of MSI-H tumors (Fig. 3A, top left) MMR-1 occurs with high relative contribution in the majority of MSI-H tumors, whereas MMR-2 and MMR-3 contributions are generally smaller and more variable across these tumors (Fig. 3A; Supplemental Fig. $\mathrm{S} 5 \mathrm{~A}, \mathrm{~B})$. However, the most severely hypermutated samples are largely described by a single signature (Fig. 3A, MMR-2 in purple, MMR-3 in orange). Minor contributions of MMR-2 and MMR-3 in other tumors may be due to the tendency of the signature calculation method to extract signatures predominantly from the most extreme cases and imparting them on other samples. We considered that MMR-2 and MMR-3 might reflect different substrate specificities of MMR complexes arising from an inactivation of unique subunits of MutS $\alpha$, MutS $\beta, \operatorname{MutL} \alpha$, or MutL $\gamma$ heterodimers. Investigating MMR gene mutations and methylation status in MSI tumor samples, we only observed few cases of MSH6 (MutS $\alpha$ ), MSH3 (MutS $\beta$ ), PMS2 (MutL $\alpha$ ), PMS1 (MutL $\beta$ ), and MLH3 (MutL $\gamma$ ) high-impact mutations often in combination with inactivation of other MMR genes (Supplemental Material; Supplemental Table S3). We observed an increased number of mu- tations assigned to the Clock-1 signature in human MMR-deficient samples (Fig. 3A,E). The Clock-1/COSMIC signature 1 is thought to reflect spontaneous $5 \mathrm{meC}$ deamination and its conversion to thymine, a mutational process that is thought to be active in all tissues and that correlates with the age at the time of cancer diagnosis (Alexandrov et al. 2013a). Our data suggest a role of MMR in the repair of 5meC deamination-induced mismatches (Bellacosa 2001; Tricarico et al. 2015; Grin and Ishchenko 2016). Notably the most frequent MMR-associated COSMIC signature, Signature 6, shows high rates of $\mathrm{C}>\mathrm{T}$ mutations in an NCG context possibly reflecting imperfect delineation of the underlying mutational processes.

A second sample cluster is represented by six tumors (Fig. 3A, bottom right) with most of their mutations falling within the POLE signature (brown). Consistently, these samples also carry pathogenic POLE mutations (Supplemental Table S4). Another cluster is formed by a subset of stomach cancer samples carrying a 17-like signature (Fig. 3A, bottom left). Four tumor samples outside of these clusters and dispersed over the similarity map are MSI. These tumors may have acquired MMR deficiency very late in their development. 
A

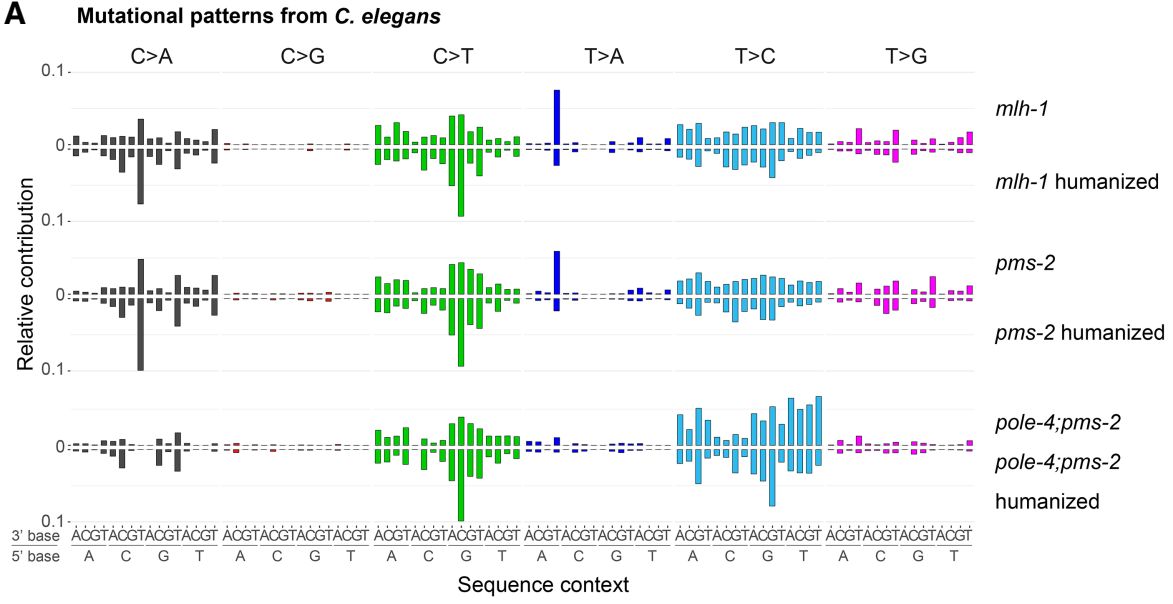

B Trinucleotide context comparison

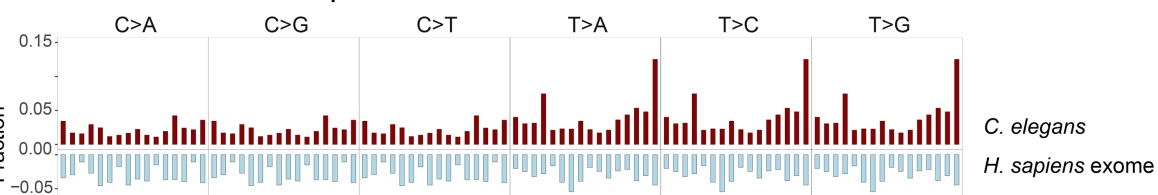

$-0.15$

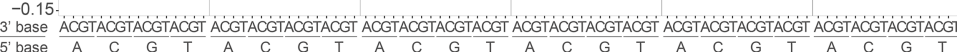

$\begin{array}{rllllllllllllllll} & & & \end{array}$

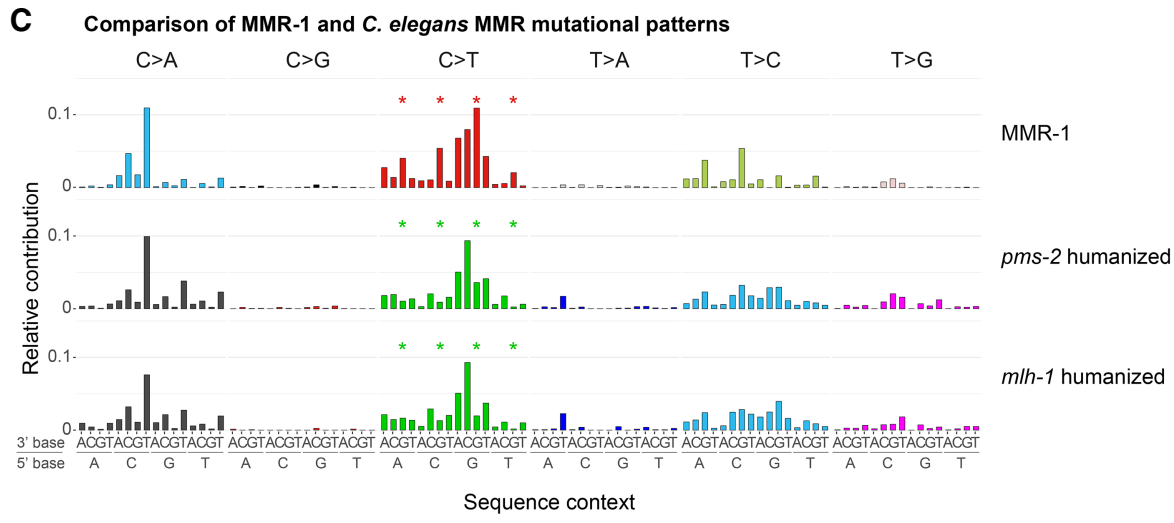

Figure 4. Mutational patterns derived from $C$. elegans MMR mutants and their comparison to the de novo human signature MMR-1. (A) Base substitution patterns of C. elegans $\mathrm{mlh}-1$, pms-2, and pole-4; pms-2 mutants and their corresponding humanized versions (mirrored). (B) Relative abundance of trinucleotides in the $C$. elegans genome (red) and the human exome (light blue). (C) MMR-1 base substitution signature compared to pms-2 and $\mathrm{m} / \mathrm{h}-1$ mutational patterns adjusted to human whole-exome trinucleotide frequency. Stars indicate the difference in C > T transitions at CpG sites, which occur at lower frequency in C. elegans.

To compare human and C. elegans MMR footprints, we first determined mutational patterns from mlh-1 and pms-2 single mutants as well as from the pole-4; pms-2 double mutant (Methods). Mutational patterns of $m l h-1$ and $p m s-2$ mutants were nearly identical with a cosine similarity of 0.97 (Fig. 4A, top panels). In contrast the pole-4; pms-2 mutational pattern showed a different relative contribution of $\mathrm{C}>\mathrm{T}$ and $\mathrm{T}>\mathrm{C}$ mutations (Fig. 4A, top panels) and displayed a cosine similarity to $m l h-1$ and $p m s-2$ below 0.71 (Supplemental Fig. S6C). We next adjusted for the difference in trinucleotide frequencies in the C. elegans genome and the human exome (Fig. 4A, bottom panels, 4B). Comparison of $C$. elegans MMR patterns with known cancer signatures showed the highest similarity of 0.77 to COSMIC signature 20 (Table 1; Supplemental Fig. S7). Of the three human MMR-associated de novo signatures, only MMR-1 displayed similarity to C. elegans MMR substitution patterns with a cosine similarity of 0.84 to pms- 2 and of 0.81 to $m l h-1$ (Table 1 ; Fig. 4C). A notable difference in the $C$. elegans pms-2 and mlh-1 patterns compared to the MMR-1 signature are a reduced level of $\mathrm{C}>\mathrm{T}$ mutations in NCG contexts (Fig. 4C, stars) and a high frequency of T > A mutation in an ATT context. The first is likely due to the lack of spontaneous deamination of 5methyl$\mathrm{C}$, a base modification that is absent in C. elegans (Greer et al. 2015); the latter is likely due to a higher relative frequency of poly(A) and poly(T) homopolymers in the $C$. elegans genome versus the human exome (Fig. 2A; Supplemental Fig. S4A). Excluding $\mathrm{C}>\mathrm{T}$ s in NCG contexts from the analysis increases the cosine similarity of MMR-1 up to 0.92 for pms-2 and up to 0.90 for $m l h-1$ patterns and reduces the uncertainty in similarity scores (Supplemental Material; Supplemental Fig. S6D). The similarity between C. elegans MMR and the human MMR-1 mutational footprints is further supported by the concurrent presence of a large number of 1-bp indels. None of the human signatures showed notable similarity to the pole-4; pms-2 mutation pattern.

\section{Discussion}

MMR-deficient tumors have among the highest mutation rates across cancer types. In line with this observation, we observed a dramatic increase in base substitutions and indels in C. elegans mlh-1 and pms-2 mutants. This mutation rate is only surpassed by that of the pole-4; pms-2 double mutant in which mutation rates are further increased two- to threefold. Genome maintenance is highly efficient as evidenced by a wild-type $C$. elegans mutation rate in the order of $8 \times$ $10^{-10}$ per base and cell division. It thus appears that DNA repair pathways act highly redundantly, and it may require the combined deficiency of multiple DNA repair pathways to trigger excessive mutagenesis. Equally, a latent defect in DNA replication integrity might only become apparent in conjunction with a DNA repair deficiency. Indeed the increased mutation burden detected in the pole-4; pms-2 double mutant, while no increased mutation rate is observed in pole- 4 alone, uncovers a latent role of pole-4. It appears that replication errors occur at increased frequency in the absence of $C$. elegans pole- 4 but are effectively repaired by MMR.

Out of the signatures associated with MMR deficiency in cancer cells, only MMR- 1 is related to the mutational pattern found in

\section{Genome Research}

www.genome.org 
Table 1. Cosine similarity values for the comparison between humanized C. elegans derived MMR mutation patterns with human de novo signatures and selected COSMIC signatures (both adjusted to human whole-exome trinucleotide frequencies)

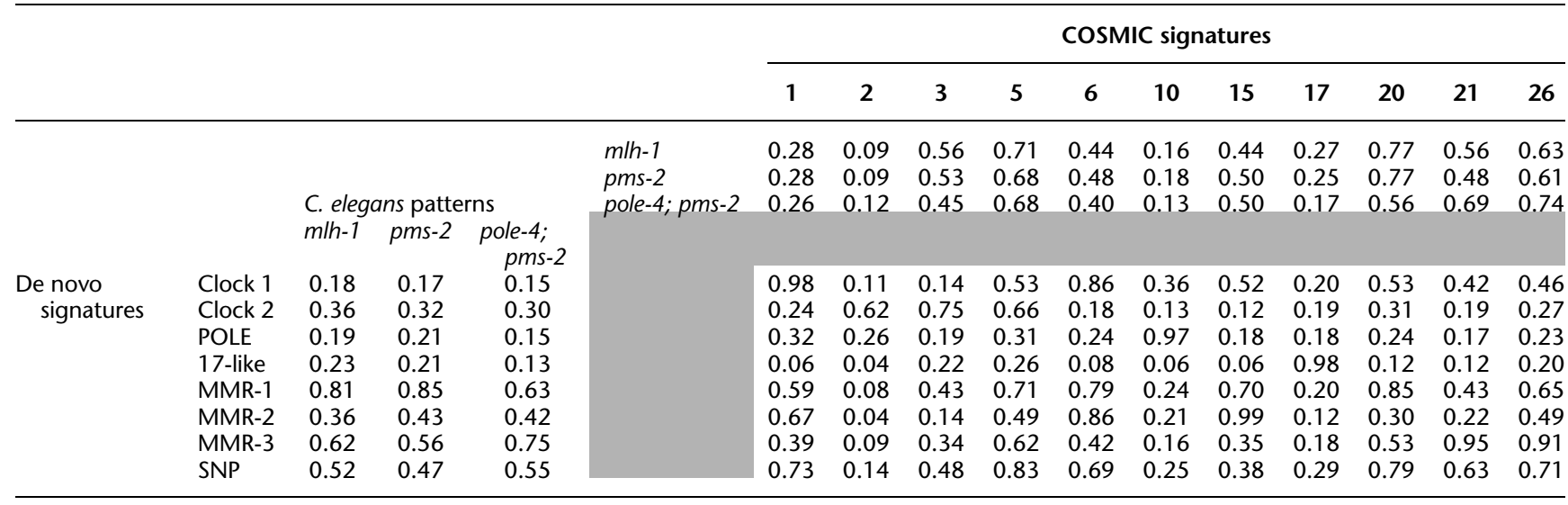

C. elegans mlh-1 and pms-2 mutants. Considering the controlled nature of the C. elegans experiment, we postulate that MMR-1 reflects a conserved mutational process of DNA replication repaired by MMR. Consistent with this, we find that MMR-1 activity is closely linked to MSI status, an established indicator for mismatch repair deficiency. In cases of hypermutation, we suggest that akin to the pole-4; pms-2 double mutant, mutational footprints can be attributed to the failed repair of lesions originating from mutations in DNA repair or DNA replication genes. For instance, in MMR-defective lines also carrying POLE catalytic subunit mutations, the mutational landscape is overwhelmed by the POLE signature (Shlien et al. 2015). Likewise it appears possible that the MMR-2 and MMR-3 signatures could be attributed to other mutational processes, which are repaired by MMR and lead to hypermutation under MMR deficiency. Overall, MMR-1 seemingly reflects a "basal" mutational process in both humans and C. elegans. In addition, human MMR deficiency also includes an element of failing to repair lesions arising from $\mathrm{CpG}$ deamination and leading to $\mathrm{C}>\mathrm{T}$ mutations, a process absent in C. elegans due to the lack of cytosine methylation. The associated human signature, "Clock-1," together with MMR-1 explains the majority of mutations occurring in MMR-defective cancers not apparently affected by hypermutation.

Matching mutational signatures to DNA repair deficiency has a tremendous potential to stratify cancer therapy tailored to DNA repair deficiency. This approach appears advantageous over genotyping marker genes, as mutational signatures provide a read-out for cellular repair deficiency associated with either genetic or epigenetic defects. Following on from our study, we expect that analyzing DNA repair-defective model organisms and human cell lines, alone or in conjunction with defined genotoxic agents, will contribute to a more precise definition of mutational signatures occurring in cancer genomes and to establishing the etiology of these signatures.

\section{Methods}

\section{C. elegans maintenance and propagation}

C. elegans mutants pole-4(tm4613) II, pms-2(ok2529) V, and mlh-1 (ok1917) III were backcrossed six times against the wild-type N2 reference strain TG1813 (Meier et al. 2014). pole-4 II; pms-2 V double mutants were generated as described in Supplemental Material. Strains were grown for 10 or 20 generations, and genomic DNA was prepared as described (Supplemental Material; Meier et al. 2014).

\section{DNA sequencing, variant calling, and post-processing}

Illumina sequencing, variant calling, and post-processing filters were performed as described (Meier et al. 2014) with the following adjustments. WBcel235.74.dna.toplevel.fa was used as the reference genome (http://ftp.ensembl.org/pub/release-74/fasta/ caenorhabditis_elegans/dna/). Alignments were performed with BWA-MEM, and mutations were called using CaVEMan, Pindel, and BRASSII (Ye et al. 2009; Stephens et al. 2012), each available at https://github.com/cancerit. Post-processing of mutation calls was performed across a large data set of 2202 sequenced samples using filter conditions as described (Supplemental Material; Meier et al. 2014). An additional filtering step using deepSNV (https://bioconductor.org/packages/release/bioc/html/deepSNV. html) was used to correct for wrongly called base substitutions, events due to algorithm-based sequence misalignment of ends of sequence reads covering 1-bp indels (Fig. 1F).

\section{Estimating mutation rates}

Mutation rates were calculated using maximum likelihood methods, assuming 15 cell divisions per generation (Meier et al. 2014) and considering that mutations have a $25 \%$ chance to be lost, a $50 \%$ chance to be transmitted as heterozygous, and a $25 \%$ chance to become homozygous, thus becoming fixed in the line during each round of $C$. elegans self-fertilization. Wild-type, pole-4, and pms-2 mutation rates were calculated from mutations observed across F10 and F20 generations.

\section{Analysis of homopolymer sequences in C. elegans and human cancer samples}

Homopolymers, dinucleotide, and trinucleotide runs encoded in the C. elegans genome, defined here as repetitive DNA regions with a consecutive number of identical bases or repeated sequence of $n \geq 4$, were identified from the reference genome WBcel235.74 using an in-house script (Supplemental Data Analysis Scripts; https:// github.com/gerstung-lab/MMR) based on $\mathrm{R}$ packages Biostrings (http://www.bioconductor.org/packages//2.7/bioc/html/Biostrings. html, R package version 2.42.2) and GenomicRanges (https:// bioconductor.org/packages/release/bioc/html/GenomicRanges. html). Overall, 3,433,785 homopolymers, 25,126 dinucleotide, and 7615 trinucleotide repeats were identified. Matching the genomic position of 1-bp indels observed in pms-2 and pole-4; pms2 mutants to the genomic positions of homopolymers, we defined 1-bp indels present in homopolymer runs and the length of the homopolymer in which they occurred. 
Generalized additive models with a spline term were used to correlate the frequency of 1-bp indels occurring in homopolymer runs with the frequency of the respective homopolymer in the $C$. elegans genome. An average of $\sim 0.51$-bp indels arising in homopolymer sequences was observed in 101 wild-type lines of different generations, indicating the frequency with which such events might occur in wild-type or as amplification artifacts during sequencing (Supplemental Material). Consistent with COAD and STAD variant calling, homopolymer frequencies and coordinates in the human exome were calculated based on the GRCh37 human reference genome build and the coordinates of well-covered fractions of whole-exome sequencing regions as reported by Agilent SureSelect V5 Human All Exon (https://earray.chem. agilent.com/earray/). Overall, we identified 976,390 homopolymers in the human exome, which ranged from 4 to $35 \mathrm{bp}$ in length (Supplemental Fig. S4A). A more recent genome build, GRCh38, does not differ in the composition of coding regions. Therefore the analysis of homopolymer frequencies is valid using both assembly versions.

\section{De novo signature extraction from human cancer samples}

Variant calls for whole-exome sequencing data from the colorectal adenocarcinoma (COAD-US) and gastric adenocarcinoma (STADUS) projects were taken from the ICGC database (http://icgc.org). Mutational counts and contexts were inferred from variant tables using MutationalPatterns R package (http://bioconductor.org/ packages/release/bioc/html/MutationalPatterns.html). After combining indels and substitutions into vectors of length 104, we extracted the signatures using the Brunet NMF with KL-divergence as implemented in Blokzijl et al. (2016), which is equivalent to a Poisson NMF model. The number of signatures was determined based on the saturation of both the Akaike Information Criterion (AIC) (Akaike 1973) and the residual sum of squares (RSS) (Supplemental Fig. S3A; Supplemental Material).

Similarity between signatures was calculated via cosine similarity as follows:

$$
\operatorname{similarity}(S 1, S 2)=\frac{\left\langle S_{1}, S_{2}\right\rangle}{\|S 1\| \cdot\|S 2\|},
$$

where $\langle S 1, S 2\rangle$ is a scalar product of signature vectors. When compared to 96-long substitution signatures, indels were omitted from 104-long de novo signatures.

Stochastic nearest neighbor representation ( $t$-SNE) (van der Maaten and Hinton 2008) was obtained using R package "tsne" (https://cran.r-project.org/web/packages/tsne/tsne.pdf) using the cosine similarity as distance measure between mutational profiles. In order to confirm the link between signatures MMR-1-3 and MMR deficiency, we defined MMR-deficient samples as those annotated as MSI-H (microsatellite instable-high) in TCGA Clinical Explorer (Lee et al. 2015). Relative contributions of every signature to the samples from the combined data set were tested for association with MSI/MSS status using one-tailed Wilcoxon rank-sum test. All $P$-values were adjusted for multiple testing correction using the Bonferroni procedure.

\section{Comparison of $C$. elegans mismatch repair mutation patterns to cancer signatures}

To extract the signatures of individual factors from respective C. elegans samples, we used additive Poisson model with multiple factors for every trinucleotide context and indel type and calculated maximum likelihood estimates for every signature (Supplemental Material). For comparison of C. elegans and human mutational signatures, signatures acquired in C. elegans were adjusted by multiplying the probability for 96 base substitutions by the ratio of respective trinucleotide counts observed in the human exome (GRCh37) counts precalculated in Rosenthal et al. (2016) to those in the C. elegans reference genome (WBcel235). Indels were not included in the comparative analysis as they required adjustment for both base and homopolymer content. COSMIC signatures were also adjusted to exome nucleotide counts, because they were mostly derived from whole exomes (Alexandrov et al. $2013 \mathrm{a}, \mathrm{b})$ and the comparison of de novo signatures to COSMIC is more valid in exome space. All signatures were further normalized so that the vector of probabilities sums up to 1 (Supplemental Table S5). For mutational signature comparison, a cosine similarity of 0.80 was considered a threshold for "high" similarity (Supplemental Material; Supplemental Fig. S6A,B).

\section{Data analysis}

R scripts (R Core Team 2017) used to analyze C. elegans and cancer data sets are available in Supplemental Data Analysis Scripts and on GitHub under https://github.com/gerstung-lab/MMR.

\section{Data access}

The sequencing data generated in this study have been submitted to the European Nucleotide Archive (ENA; https://www.ebi.ac.uk/ ena) under accession numbers ERP000975 and ERP004086, as detailed in Supplemental Table S6.

\section{Acknowledgments}

This work was supported by a Wellcome Trust Senior Research award to A.G. (090944/Z/09/Z) and by a Wellcome Trust Strategic Award (101126/B/13/Z). B.M., N.V.V., P.J.C., M.G., and A.G. are members of the Wellcome Trust funded COMSIG (Causes of Mutational SIGnatures) consortium. P.S. and the Data Analysis Group, Dundee, were funded by the "Wellcome Trust Technology Platform" Strategic Award 097945/Z/11/Z. We thank the Mitani laboratory funded by the National Bio-Resource Project, Japan, and the Caenorhabditis Genetics Center funded by the NIH Office of Research Infrastructure Programs (P40 OD010440) for providing strains. We also thank Steve Hubbard, University of Dundee, for help with statistics.

\section{References}

Akaike H. 1973. Information theory and an extension of the maximum likelihood principle. In 2nd International symposium of information theory, pp. 267-281, Akademiai Kiado, Budapest.

Aksenova A, Volkov K, Maceluch J, Pursell ZF, Rogozin IB, Kunkel TA, Pavlov YI, Johansson E. 2010. Mismatch repair-independent increase in spontaneous mutagenesis in yeast lacking non-essential subunits of DNA polymerase $\varepsilon$. PLoS Genet 6: e1001209.

Albertson TM, Ogawa M, Bugni JM, Hays LE, Chen Y, Wang Y, Treuting PM, Heddle JA, Goldsby RE, Preston BD. 2009. DNA polymerase $\varepsilon$ and $\delta$ proofreading suppress discrete mutator and cancer phenotypes in mice. Proc Natl Acad Sci 106: 17101-17104.

Alexandrov LB, Nik-Zainal S, Wedge DC, Aparicio SA, Behjati S, Biankin AV, Bignell GR, Bolli N, Borg A, Børresen-Dale AL, et al. 2013a. Signatures of mutational processes in human cancer. Nature 500: $415-421$.

Alexandrov LB, Nik-Zainal S, Wedge DC, Campbell PJ, Stratton MR. 2013b. Deciphering signatures of mutational processes operative in human cancer. Cell Rep 3: 246-259.

Allen DJ, Makhov A, Grilley M, Taylor J, Thresher R, Modrich P, Griffith JD. 1997. MutS mediates heteroduplex loop formation by a translocation mechanism. EMBO J 16: 4467-4476.

Baross-Francis A, Makhani N, Liskay RM, Jirik FR. 2001. Elevated mutant frequencies and increased $\mathrm{C}: \mathrm{G} \rightarrow \mathrm{T}: \mathrm{A}$ transitions in $\mathrm{Mlh} 1^{-/-}$versus $P m s 2^{-/-}$ murine small intestinal epithelial cells. Oncogene 20: 619-625.

Bellacosa A. 2001. Functional interactions and signaling properties of mammalian DNA mismatch repair proteins. Cell Death Differ 8: 1076-1092.

\section{Genome Research}

www.genome.org 
Bhattacharyya NP, Skandalis A, Ganesh A, Groden J, Meuth M. 1994. Mutator phenotypes in human colorectal carcinoma cell lines. Proc Natl Acad Sci 91: 6319-6323.

Blokzijl F, Janssen R, Van Boxtel R, Cuppen E. 2016. MutationalPatterns: an integrative $\mathrm{R}$ package for studying patterns in base substitution catalogues. bioRxiv doi: 10.1101/071761.

Bronner CE, Baker SM, Morrison PT, Warren G, Smith LG, Lescoe MK, Kane M, Earabino C, Lipford J, Lindblom A, et al. 1994. Mutation in the DNA mismatch repair gene homologue $h M L H 1$ is associated with hereditary non-polyposis colon cancer. Nature 368: 258-261.

Brouwer JR, Willemsen R, Oostra BA. 2009. Microsatellite repeat instability and neurological disease. Bioessays 31: 71-83.

The Cancer Genome Atlas Network. 2012. Comprehensive molecular characterization of human colon and rectal cancer. Nature 487: 330-337.

The Cancer Genome Atlas Research Network. 2014. Comprehensive molecular characterization of gastric adenocarcinoma. Nature 513: 202-209.

Cannavo E, Marra G, Sabates-Bellver J, Menigatti M, Lipkin SM, Fischer F, Cejka P, Jiricny J. 2005. Expression of the MutL homologue hMLH3 in human cells and its role in DNA mismatch repair. Cancer Res 65: 10759-10766.

Degtyareva NP, Greenwell P, Hofmann ER, Hengartner MO, Zhang L, Culotti JG, Petes TD. 2002. Caenorhabditis elegans DNA mismatch repair gene $m s h-2$ is required for microsatellite stability and maintenance of genome integrity. Proc Natl Acad Sci 99: 2158-2163.

Denver DR, Morris K, Kewalramani A, Harris KE, Chow A, Estes S, Lynch M, Thomas WK. 2004. Abundance, distribution, and mutation rates of homopolymeric nucleotide runs in the genome of Caenorhabditis elegans. $J$ Mol Evol 58: 584-595.

Denver DR, Feinberg S, Estes S, Thomas WK, Lynch M. 2005. Mutation rates, spectra and hotspots in mismatch repair-deficient Caenorhabditis elegans. Genetics 170: 107-113.

Drost J, van Boxtel R, Blokzijl F, Mizutani T, Sasaki N, Sasselli V, de Ligt J, Behjati S, Grolleman JE, van Wezel T, et al. 2017. Use of CRISPR-modified human stem cell organoids to study the origin of mutational signatures in cancer. Science 358: 234-238.

Drummond JT, Li GM, Longley MJ, Modrich P. 1995. Isolation of an hMSH2-p160 heterodimer that restores DNA mismatch repair to tumor cells. Science 268: 1909-1912.

Fishel R, Lescoe MK, Rao MR, Copeland NG, Jenkins NA, Garber J, Kane M, Kolodner R. 1993. The human mutator gene homolog MSH2 and its association with hereditary nonpolyposis colon cancer. Cell 75: 1027-1038.

Genschel J, Littman SJ, Drummond JT, Modrich P. 1998. Isolation of MutS $\beta$ from human cells and comparison of the mismatch repair specificities of MutS $\beta$ and MutS $\alpha$. J Biol Chem 273: 19895-19901.

Gerstung M, Beisel C, Rechsteiner M, Wild P, Schraml P, Moch H, Beerenwinkel N. 2012. Reliable detection of subclonal single-nucleotide variants in tumour cell populations. Nat Commun 3: 811.

Gerstung M, Papaemmanuil E, Campbell PJ. 2014. Subclonal variant calling with multiple samples and prior knowledge. Bioinformatics 30: 11981204.

Goellner EM, Putnam CD, Kolodner RD. 2015. Exonuclease 1-dependent and independent mismatch repair. DNA Repair 32: 24-32.

Gradia S, Subramanian D, Wilson T, Acharya S, Makhov A, Griffith J, Fishel R. 1999. hMSH2-hMSH6 forms a hydrolysis-independent sliding clamp on mismatched DNA. Mol Cell 3: 255-261.

Greer EL, Blanco MA, Gu L, Sendinc E, Liu J, Aristizábal-Corrales D, Hsu CH, Aravind L, He C, Shi Y. 2015. DNA methylation on $\mathrm{N}^{6}$-adenine in C. elegans. Cell 161: $868-878$.

Grin I, Ishchenko AA. 2016. An interplay of the base excision repair and mismatch repair pathways in active DNA demethylation. Nucleic Acids Res 44: 3713-3727.

Habraken Y, Sung P, Prakash L, Prakash S. 1996. Binding of insertion/deletion DNA mismatches by the heterodimer of yeast mismatch repair proteins MSH2 and MSH3. Curr Biol 6: 1185-1187.

Hanford MG, Rushton BC, Gowen LC, Farber RA. 1998. Microsatellite mutation rates in cancer cell lines deficient or proficient in mismatch repair. Oncogene 16: 2389-2393.

Harr B, Zangerl B, Schlötterer C. 2000. Removal of microsatellite interruptions by DNA replication slippage: phylogenetic evidence from Drosophila. Mol Biol Evol 17: 1001-1009.

Kadyrov FA, Dzantiev L, Constantin N, Modrich P. 2006. Endonucleolytic function of MutL $\alpha$ in human mismatch repair. Cell 126: 297-308.

Kadyrov FA, Holmes SF, Arana ME, Lukianova OA, O'Donnell M, Kunkel TA, Modrich P. 2007. Saccharomyces cerevisiae MutL $\alpha$ is a mismatch repair endonuclease. J Biol Chem 282: 37181-37190.

Lang GI, Parsons L, Gammie AE. 2013. Mutation rates, spectra, and genomewide distribution of spontaneous mutations in mismatch repair deficient yeast. G3 3: 1453-1465.

Lawrence M, Huber W, Pagès H, Aboyoun P, Carlson M, Gentleman R, Morgan MT, Carey VJ. 2013. Software for computing and annotating genomic ranges. PLoS Comput Biol 9: e1003118.
Lee H, Palm J, Grimes SM, Ji HP. 2015. The Cancer Genome Atlas Clinical Explorer: a web and mobile interface for identifying clinical-genomic driver associations. Genome Med 7: 112.

Lujan SA, Williams JS, Pursell ZF, Abdulovic-Cui AA, Clark AB, Nick McElhinny SA, Kunkel TA. 2012. Mismatch repair balances leading and lagging strand DNA replication fidelity. PLoS Genet 8: e1003016.

Lujan SA, Clausen AR, Clark AB, MacAlpine HK, MacAlpine DM, Malc EP, Mieczkowski PA, Burkholder AB, Fargo DC, Gordenin DA, et al. 2014 Heterogeneous polymerase fidelity and mismatch repair bias genome variation and composition. Genome Res 24: 1751-1764.

Lujan SA, Clark AB, Kunkel TA. 2015. Differences in genome-wide repeat sequence instability conferred by proofreading and mismatch repair defects. Nucleic Acids Res 43: 4067-4074.

Meier B, Cooke SL, Weiss J, Bailly AP, Alexandrov LB, Marshall J, Raine K, Maddison M, Anderson E, Stratton MR, et al. 2014. C. elegans whole-genome sequencing reveals mutational signatures related to carcinogens and DNA repair deficiency. Genome Res 24: 1624-1636.

Miyaki M, Konishi M, Tanaka K, Kikuchi-Yanoshita R, Muraoka M, Yasuno M, Igari T, Koike M, Chiba M, Mori T. 1997. Germline mutation of MSH6 as the cause of hereditary nonpolyposis colorectal cancer. Nat Genet 17: 271-272.

Nicolaides NC, Papadopoulos N, Liu B, Wei YF, Carter KC, Ruben SM, Rosen CA, Haseltine WA, Fleischmann RD, Fraser CM, et al. 1994. Mutations of two PMS homologues in hereditary nonpolyposis colon cancer. Nature 371: $75-80$.

Palles C, Cazier JB, Howarth KM, Domingo E, Jones AM, Broderick P, Kemp Z, Spain SL, Guarino E, Salguero I, et al. 2013. Germline mutations affecting the proofreading domains of POLE and POLD1 predispose to colorectal adenomas and carcinomas. Nat Genet 45: 136-144.

Papadopoulos N, Nicolaides NC, Wei YF, Ruben SM, Carter KC, Rosen CA, Haseltine WA, Fleischmann RD, Fraser CM, Adams MD, et al. 1994 Mutation of a mutL homolog in hereditary colon cancer. Science 263: $1625-1629$.

Prolla TA, Baker SM, Harris AC, Tsao JL, Yao X, Bronner CE, Zheng B, Gordon M, Reneker J, Arnheim N, et al. 1998. Tumour susceptibility and spontaneous mutation in mice deficient in Mlh1, Pms1 and Pms2 DNA mismatch repair. Nat Genet 18: 276-279.

R Core Team. 2017. R: a language and environment for statistical computing. R Foundation for Statistical Computing, Vienna, Austria. https://www.Rproject.org/.

Robinson JT, Thorvaldsdóttir H, Winckler W, Guttman M, Lander ES, Getz G, Mesirov JP. 2011. Integrative genomics viewer. Nat Biotechnol 29: 24-26.

Rosenthal R, McGranahan N, Herrero J, Taylor BS, Swanton C. 2016. deconstructSigs: Delineating mutational processes in single tumors distinguishes DNA repair deficiencies and patterns of carcinoma evolution. Genome Biol 17: 31.

Shlien A, Campbell BB, de Borja R, Alexandrov LB, Merico D, Wedge D, Van Loo P, Tarpey PS, Coupland P, Behjati S, et al. 2015. Combined hereditary and somatic mutations of replication error repair genes result in rapid onset of ultra-hypermutated cancers. Nat Genet 47: 257-262.

Stephens PJ, Tarpey PS, Davies H, Van Loo P, Greenman C, Wedge DC, NikZainal S, Martin S, Varela I, Bignell GR, et al. 2012. The landscape of cancer genes and mutational processes in breast cancer. Nature 486: 400-404.

Strand M, Prolla TA, Liskay RM, Petes TD. 1993. Destabilization of tracts of simple repetitive DNA in yeast by mutations affecting DNA mismatch repair. Nature 365: 274-276.

Supek F, Lehner B. 2015. Differential DNA mismatch repair underlies mutation rate variation across the human genome. Nature 521: 81-84.

Tijsterman M, Pothof J, Plasterk RH. 2002. Frequent germline mutations and somatic repeat instability in DNA mismatch-repair-deficient Caenorhabditis elegans. Genetics 161: 651-660.

Tricarico R, Cortellino S, Riccio A, Jagmohan-Changur S, Van der Klift $\mathrm{H}$ Wijnen J, Turner D, Ventura A, Rovella V, Percesepe A, et al. 2015 Involvement of $M B D 4$ inactivation in mismatch repair-deficient tumorigenesis. Oncotarget 6: 42892-42904.

van der Maaten L, Hinton G. 2008. Visualizing high-dimensional data using t-SNE. J Mach Learn Res 9: 2579-2605.

Yang Y, Karthikeyan R, Mack SE, Vonarx EJ, Kunz BA. 1999. Analysis of yeast pms $1, m s h 2$, and $m l h 1$ mutators points to differences in mismatch correction efficiencies between prokaryotic and eukaryotic cells. Mol Gen Genet 261: 777-787.

Yao X, Buermeyer AB, Narayanan L, Tran D, Baker SM, Prolla TA, Glazer PM, Liskay RM, Arnheim N. 1999. Different mutator phenotypes in Mlh1versus Pms2-deficient mice. Proc Natl Acad Sci 96: 6850-6855.

Ye K, Schulz MH, Long Q, Apweiler R, Ning Z. 2009. Pindel: a pattern growth approach to detect break points of large deletions and medium sized insertions from paired-end short reads. Bioinformatics 25: 2865-2871.

Received June 27, 2017; accepted in revised form January 2, 2018. 


\section{Corrigenda}

Genome Research 28: 666-675 (2018)

Corrigendum: Mutational signatures of DNA mismatch repair deficiency in $C$. elegans and human cancers

Bettina Meier, Nadezda V. Volkova, Ye Hong, Pieta Schofield, Peter J. Campbell, Moritz Gerstung, and Anton Gartner

The authors would like to correct an error in the link to the sequencing data. The Data access section in the text has been corrected as follows and has been updated online:

The sequencing data generated in this study have been submitted to the European Nucleotide Archive (ENA; https://www.ebi.ac.uk/ena) under accession numbers ERP000975 and ERP004086, as detailed in Supplemental Table S6.

Additionally, Supplemental Tables S1-S6 were inadvertently excluded from the initial publication of this article. Please note that the Supplemental Tables have now been published online in the Additional Supplemental Material.

The authors apologize for the inconvenience.

doi: $10.1101 /$ gr.255596.119

Genome Research 24: 629-638 (2014)

Corrigendum: A long-term demasculinization of $\mathrm{X}$-linked intergenic noncoding RNAs in Drosophila melanogaster

Ge Gao, Maria D. Vibranovski, Li Zhang, Zheng Li, Min Liu, Yong E. Zhang, Xinmin Li, Wenxia Zhang, Qichang Fan, Nicholas W. VanKuren, Manyuan Long, and Liping Wei

The name of the fifth author was originally misspelled in the author line of this article. Please note the correct spelling above as Min Liu.

doi: $10.1101 /$ gr.255315.119 


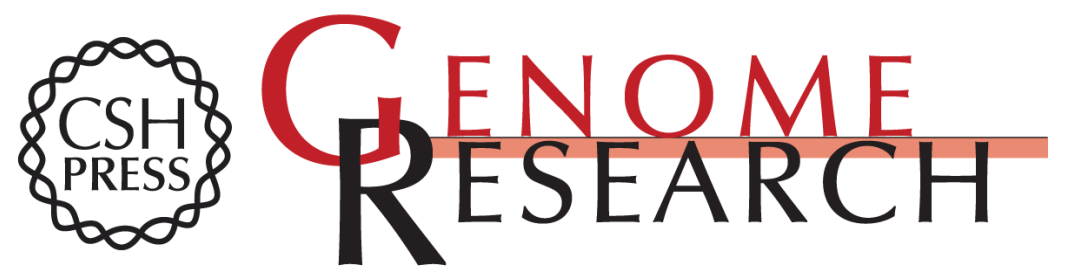

\section{Mutational signatures of DNA mismatch repair deficiency in $C$. elegans and human cancers}

Bettina Meier, Nadezda V. Volkova, Ye Hong, et al.

Genome Res. 2018 28: 666-675 originally published online April 10, 2018

Access the most recent version at doi:10.1101/gr.226845.117

\section{Supplemental Material \\ Related Content \\ References
Creative
Commons
License \\ Open Access}

\section{Email Alerting} Service
http://genome.cshlp.org/content/suppl/2018/04/10/gr.226845.117.DC1 http://genome.cshlp.org/content/suppl/2019/08/21/gr.226845.117.DC2

Corrigendum: Mutational signatures of DNA mismatch repair deficiency in $\mathrm{C}$. elegans and human cancers Bettina Meier, Nadezda V. Volkova, Ye Hong, et al.

Genome Res. September , 2019 29: 1566_2

This article cites 56 articles, 16 of which can be accessed free at: http://genome.cshlp.org/content/28/5/666.full.html\#ref-list-1

Articles cited in:

http://genome.cshlp.org/content/28/5/666.full.html\#related-urls

Freely available online through the Genome Research Open Access option.

This article, published in Genome Research, is available under a Creative Commons License (Attribution 4.0 International), as described at http://creativecommons.org/licenses/by/4.0/.

Receive free email alerts when new articles cite this article - sign up in the box at the top right corner of the article or click here.

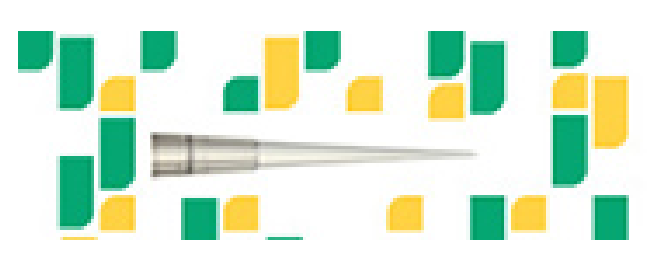

Focused on your science.

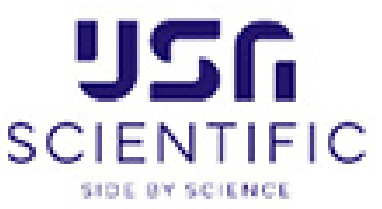

To subscribe to Genome Research go to:

https://genome.cshlp.org/subscriptions

(C) 2018 Meier et al.; Published by Cold Spring Harbor Laboratory Press 